\title{
Conflitos na prática profissional em ambientes de urgência e emergência
}

\author{
Conflicts in professional practice at urgent and emergency services
}

Conflictos en la práctica profesional en servicios de urgencia y emergencia

Layane Nunes de Lima ${ }^{1}$, Eurípedes Gil de França ${ }^{2 *}$, Rachel Mola ${ }^{1}$, Lusineide Carmo Andrade de Lacerda ${ }^{1}$, Leonel Batista de Lima Neto ${ }^{3}$, Amanda Regina da Silva Góis ${ }^{1}$.

\section{RESUMO}

Objetivo: Identificar as circunstâncias que resultam no ou contribuem para o surgimento de conflitos na prática profissional em ambientes de urgência e emergência, os sujeitos envolvidos e as consequências desses conflitos. Métodos: Revisão integrativa, realizada em outubro de 2020 nas bases de dados MEDLINE, LILACS e BDEnf. Buscaram-se referências entre os anos de 2015 e 2020. Selecionaram-se 10 artigos para as análises. Resultados: As circunstâncias identificadas foram dificuldades relacionadas à comunicação, problemas de estrutura e/ou ambiente laboral das instituições e a sobrecarga de trabalho. Os sujeitos envolvidos foram médicos, enfermeiros, outros profissionais da equipe de saúde e familiares. Quanto às consequências dos conflitos, identificaram-se problemas psicológicos, sentimento de reclusão, sufocamento laboral e impotência. Verificou-se que a enfermagem tende a estar mais vulnerável à ocorrência de conflitos. Considerações finais: Conflitos são inerentes à convivência humana e precisam ser administrados a fim de evitar o comprometimento da integralidade da assistência à saúde. Caso os conflitos sejam bem gerenciados, poderão repercutir positivamente para melhorias no ambiente de trabalho.

Palavras-chave: Conflito psicológico, Emergências, Estresse profissional, Relações interpessoais, Assistência de enfermagem.

\begin{abstract}
Objective: To identify the circumstances that result in or contribute to the emergence of conflicts in professional practice at urgent and emergency services, the subjects involved, and the consequences of these conflicts. Methods: Integrative review, carried out in October 2020 by searching the databases MEDLINE, LILACS, and BDEnf. References were sought between the years 2015 and 2020. Ten articles were selected for analysis. Results: The circumstances identified were difficulties related to communication, issues in structure and/or workplace of institutions, and work overload. The subjects involved were physicians, nurses, other health team professionals, and family members. As for the consequences of conflicts, psychological issues, seclusion feelings, labor suffocation, and powerlessness were identified. It was found that nursing tends to be more vulnerable to the occurrence of conflicts. Final remarks: Conflicts are inherent to human coexistence and they need to be tackled to prevent health care from being affected. If conflicts are well managed, they can have positive repercussions on improvements in the workplace.
\end{abstract}

Keywords: Psychological conflict, Urgent and emergency care, Professional stress, Interpersonal relationships, Nursing care.

1 Universidade de Pernambuco (UPE), Petrolina - PE.

2 Universidade de Pernambuco (UPE), Recife - PE. *E-mail: euripedesgf@gmail.com

${ }^{3}$ Universidade Federal do Vale do São Francisco (HU-UNIVASF), Petrolina - PE. 


\section{RESUMEN}

Objetivo: Identificar las circunstancias que resultan en o contribuyen a la aparición de conflictos en la práctica profesional en servicios de urgencia y emergencia, los sujetos involucrados, y las consecuencias de estos conflictos. Métodos: Revisión integradora, realizada en octubre de 2020 mediante búsqueda en las bases de datos MEDLINE, LILACS, y BDEnf. Se buscaron referencias entre los años 2015 y 2020. Se seleccionaron 10 artículos para análisis. Resultados: Las circunstancias identificadas fueron dificultades relacionadas con la comunicación, problemas con la estructura, y/o ambiente de trabajo en las instituciones y sobrecarga de trabajo. Los sujetos involucrados fueron médicos, enfermeros, otros profesionales del equipo de salud, y familiares. En cuanto a las consecuencias de los conflictos, se identificaron problemas psicológicos, sentimientos de reclusión, asfixia laboral, e impotencia. Se encontró que la enfermería tiende a ser más vulnerable a la ocurrencia de conflictos. Consideraciones finales: Conflictos son inherentes a la convivencia humana y necesitan ser gestionados para evitar que la atención de salud se vea comprometida. Si los conflictos se gestionan bien, pueden tener repercusiones positivas para la mejora del entorno laboral.

Palabras clave: Conflicto psicológico, Atención de urgencia y emergencia, Estrés profesional, Relaciones interpersonales, Atención de enfermería.

\section{INTRODUÇÃO}

As urgências e emergências hospitalares requerem dos profissionais de saúde habilidades para estabelecerem uma rotina harmoniosa de convívio visto que esses profissionais, frequentemente, estão em contato com uma diversidade de pessoas, sejam elas pacientes, familiares e outros membros da equipe de saúde, cada uma com sua particular cosmovisão, crenças, perspectivas, posicionamentos ideológicos. Além disso, dada a rotina dos serviços, o ambiente das urgências e emergências é considerado agitado e estressante, e há registros de que a convivência profissional ocorre em meio a situações de estresse e propensas ao surgimento de conflitos (PICOLI RP, et al., 2016).

Conflitos dizem respeito às discordâncias entre pessoas ou grupos de pessoas e resultam das divergências de ideias, valores ou sentimentos, podendo concorrer para a ruptura da ordem e para a vivência de experiências negativas (BESERRA EP, et al., 2018).

São diversas as situações que contribuem para o surgimento dos conflitos, tais como condições laborais desfavoráveis, demanda elevada, superlotação do serviço, sobrecarga de trabalho, exigência física do profissional e pressões relacionadas às cobranças (FERNANDES RTP e COELHO MJ, 2013; FROTA CA, et al., 2021).

Pesquisas sugerem que os conflitos resultam em elevadas taxas de absenteísmo, alta rotatividade de profissionais no serviço, aumento das queixas profissionais, diminuição do comprometimento no trabalho, esgotamento, oneração do sistema de saúde e prejuízos orçamentários consideráveis (VIVAR CG, 2006; YARBROUGH S, et al., 2016; LANZ JJ e BRUK-LEE V, 2017). Em estudo realizado no Brasil, verificou-se que a existência de conflito era uma característica do ambiente de urgência e emergência e gerava dificuldades para o desempenho das funções profissionais (DUARTE MLC, et al., 2018).

As relações interpessoais harmoniosas são fundamentais no contexto do trabalho multidisciplinar visto que do sucesso desta interação dependem a qualidade do cuidado oferecido ao paciente para o alcance do objetivo maior: a melhora do quadro clínico e subsequente alta hospitalar da pessoa internada. Pesquisas recomendam que os serviços de saúde devam estar aptos a gerenciar os conflitos existentes e identificar situações potenciais para o seu surgimento. Entende-se, portanto, que estudar sobre os conflitos poderá contribuir para a compreensão dos motivos pelos quais eles ocorrem e, assim, fomentar a produção de resultados que auxiliem na elaboração de estratégias de enfrentamento e que deem suporte para a tomada de decisão (BESERRA EP, et al., 2018; BAŞOĞUL C e ÖZGÜR G, 2016).

Este estudo teve como objetivo identificar, na literatura científica, as circunstâncias que resultam ou contribuem para o surgimento de conflitos na prática profissional em ambientes de urgência e emergência, assim como identificar os sujeitos envolvidos e as consequências resultantes desses conflitos. 


\section{MÉTODOS}

Estudo de revisão integrativa, com fins descritivo, exploratório e abordagem qualitativa. Para tanto, utilizouse da tradicional recomendação de Whittemore R e Knalf K (2005) que atualizaram o método da revisão, sugerindo cinco passos sequenciais para a sua realização: 1) identificação do problema de pesquisa, 2) busca na literatura, 3) avaliação dos dados, 4) análise dos dados e 5) apresentação dos dados. Na primeira etapa, identificou-se o problema por meio das seguintes questões norteadoras da pesquisa: que circunstâncias resultam ou contribuem para o surgimento de conflitos envolvendo profissionais em ambiente de urgência e emergência? Quais os sujeitos envolvidos nesses conflitos? E quais as consequências resultantes desses conflitos gerados?

A segunda etapa da revisão diz respeito a definição da estratégia e realização da busca de publicações em bases de dados e periódicos. Definiram-se os termos de busca, conforme os Descritores em Ciências da Saúde/Medical Subject Headings (DeCS/MeSH), e estes foram manipulados de acordo com a seguinte estratégia, na modalidade avançada: "enfermagem AND conflito psicológico", "enfermagem AND relações interpessoais AND conflito psicológico". A busca foi realizada nas bases de dados Medical Literature Analysis and Retrieval System Online (MEDLINE), Literatura Latino-Americana em Ciências da Saúde (LILACS) e Base de dados em Enfermagem (BDEnf).

Os critérios de inclusão para seleção dos artigos foram os seguintes: publicações que abordassem os conflitos em ambiente de urgência e emergência, nos idiomas inglês, espanhol e português, indexados entre os anos de 2015 e 2020 e disponíveis na íntegra. Foram excluídos artigos do tipo editorial, revisões, monografias, dissertações e teses.

$\mathrm{Na}$ terceira etapa procedeu-se a avaliação dos resultados de busca, seleção e avaliação dos artigos. Do critério de busca anteriormente definido, resultaram 157 publicações (MEDLINE, $n=133$; LILACS, $n=11$; BDEnf, $n=13$ ). Inicialmente foram realizadas as leituras dos títulos e resumos e, destas, excluíram-se 128 artigos: 97 destes não tratavam das circunstâncias que resultam ou contribuem para o surgimento de conflitos em ambiente de urgência e emergência, 14 não estavam relacionados à atuação de profissionais em ambiente de urgência e emergência, e um (1) estava dentro do escopo dos tipos de publicações excluídas; 16 eram artigos repetidos entre as bases. Restaram 29 artigos para a leitura integral. Após esta leitura, julgou-se que 19 dos trabalhos fugiam à proposta definida nas questões norteadoras desta revisão e, por isso, também foram excluídos. A amostra final foi composta por 10 artigos (Figura 1). 
Figura 1 - Fluxograma das etapas da revisão integrativa.
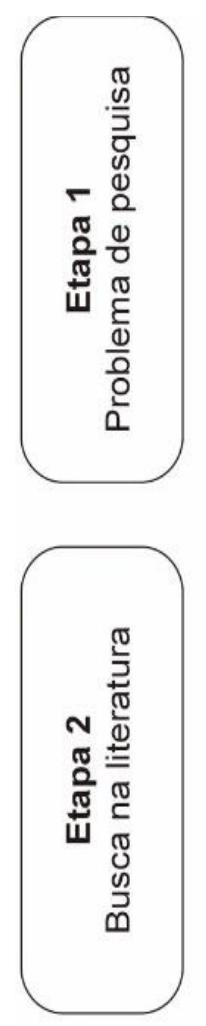

Questões norteadoras:

Que circunstâncias resultam ou contribuem para o surgimento de conflitos envolvendo profissionais em ambiente de urgência e emergência? Quais os sujeitos envolvidos nesses conflitos? E quais as consequências resultantes desses conflitos gerados?

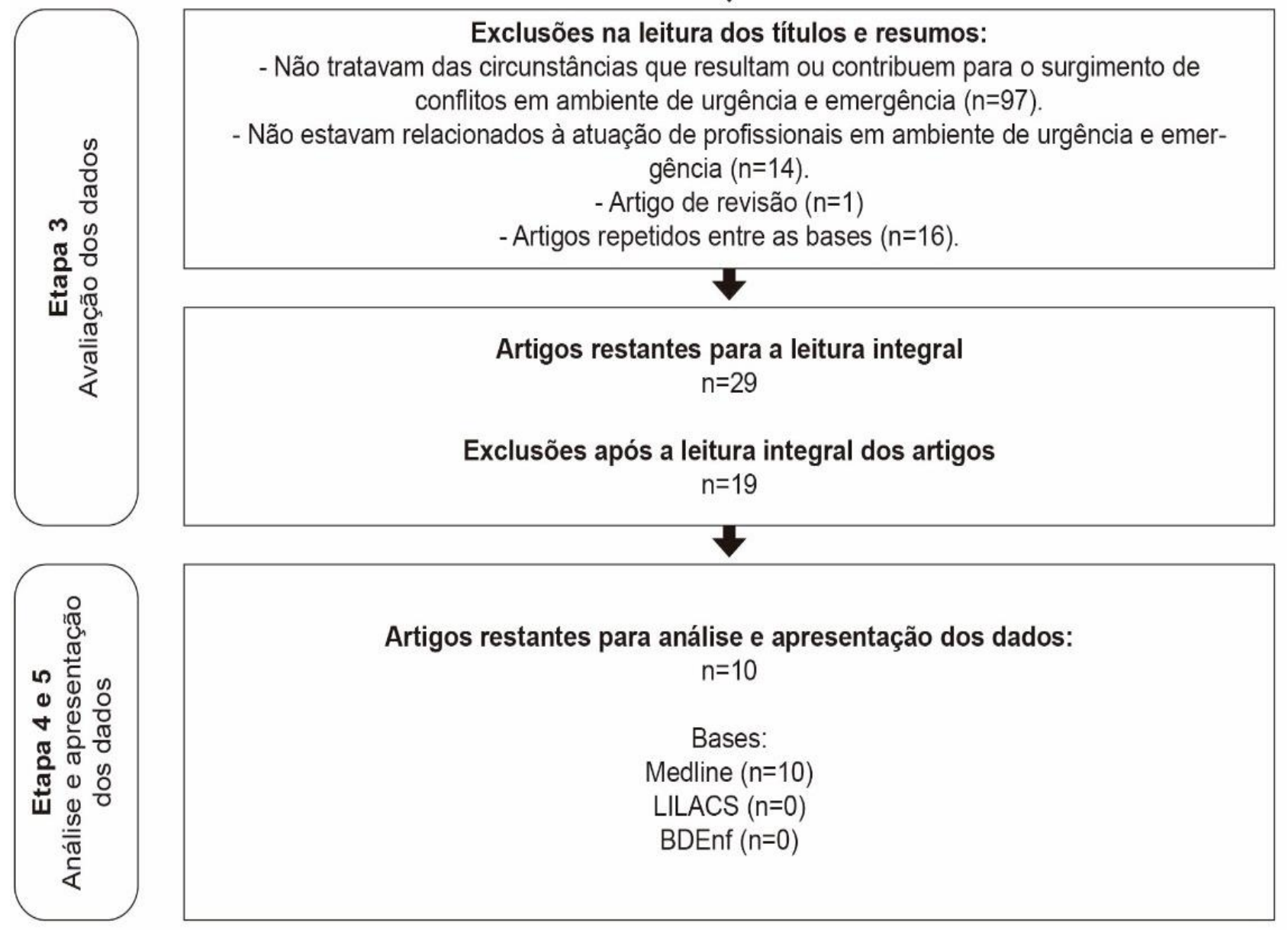

Fonte: Lima LN, et al., 2021. 
$\mathrm{Na}$ quarta etapa procedeu-se à análise dos dados. Após a leitura na íntegra, os artigos foram fichados e as informações pertinentes destacadas e sintetizadas em planilha. As variáveis numéricas (ano de publicação, idioma e país de publicação) foram organizadas para uma breve exposição quantitativa. Quanto às variáveis categóricas, foram sintetizadas categorias temáticas para a exposição e discussão qualitativa.

Na quinta etapa realizou-se a exposição dos dados e os artigos selecionados foram, inicialmente, descritos quantitativamente, em conformidade com as suas variáveis numéricas. Em seguida, os artigos foram registrados individualmente, em uma matriz de coleta, por ordem crescente de ano de publicação, denominados de "ID" (identificação) seguido de algarismo arábico (ID1 a ID11), destacando os autores, periódicos, objetivos, métodos utilizados e resultados. Finalmente, apresentaram-se os resultados qualitativos e foram discutidas as categorias encontradas.

\section{RESULTADOS}

Dez artigos foram elegíveis para a análise, síntese e apresentação dos resultados. A maior parte deles foi publicada no ano de 2015, tendo sido observada uma tendência decrescente de publicação nos anos seguintes. Todos estavam em idioma inglês e a Inglaterra predominou quanto ao país que mais publicou a respeito do tema, apesar de não necessariamente as pesquisas terem sido realizadas neste país. Sessenta por cento da amostra foi de trabalhos de abordagem quantitativa e 3.455 profissionais foram envolvidos, média de 575,8 profissionais por estudo. Já entre os estudos qualitativos, 199 sujeitos foram investigados, média de 49,75 por estudo. Todos os artigos aplicaram o corte transversal de coleta e exposição de dados de forma descritiva, e dentre os qualitativos a estratégia de coleta principal foi a entrevista.

Em relação aos conflitos na prática profissional, em ambientes de urgência e emergência, houve predomínio de pesquisas na área da enfermagem. Os dados foram agrupados em três categorias temáticas, de acordo com a proposta das questões norteadoras da pesquisa: circunstâncias geradoras de conflitos, sujeitos envolvidos, e consequências (dos conflitos).

As principais circunstâncias geradoras de conflitos estiveram relacionadas à comunicação ou problemas relacionados à comunicação (ID1, ID2, ID3 e ID6), estrutura e/ou ambiente laboral das instituições (ID3, ID6 e ID10) e sobrecarga de trabalho (ID1 e ID8). Em relação aos sujeitos envolvidos, verificaram-se conflitos entre enfermeiros e familiares (ID3, ID5, ID7, ID8, ID9 e ID10), entre enfermeiros e médicos (ID1, ID2, ID3, ID5 e ID10) e entre os próprios profissionais da equipe de enfermagem (ID2, ID6 e ID10), ainda que os motivos pelos quais estes conflitos ocorrem não esteja devidamente esclarecido. Em relação às consequências dos conflitos, observou-se que a enfermagem tende a sofrer mais com os conflitos na prática profissional, e estes afetam a saúde psicológica destes profissionais (ID7). Além disso, observaram-se casos de sentimento de reclusão, sufocamento laboral e impotência (ID1, ID3, ID4 e ID7). Todavia, verificou-se que, caso os conflitos sejam bem gerenciados, poderão repercutir positivamente para melhorias no ambiente de trabalho (ID2) (Quadro 1). 
Quadro 1 - Caracterização dos estudos selecionados para a revisão.

\begin{tabular}{|c|c|c|c|c|c|}
\hline ID & $\begin{array}{l}\text { Autor, ano e país } \\
\text { de publicação }\end{array}$ & Periódico & Objetivos & Método utilizado & Resultados \\
\hline 1 & $\begin{array}{l}\text { Moreland JJ e } \\
\text { Apker J (2015) } \\
\quad \text { Inglaterra }\end{array}$ & $\begin{array}{c}\text { Health } \\
\text { Communication }\end{array}$ & $\begin{array}{l}\text { Explorar as formas pelas quais os } \\
\text { conflitos de comunicação e o estresse } \\
\text { comunicativo são vivenciados e } \\
\text { tolerados na enfermagem; e } \\
\text { compreender como os enfermeiros } \\
\text { (mal)gerenciam, por meio do diálogo, } \\
\text { conflitos e estresses. }\end{array}$ & $\begin{array}{l}\text { Qualitativo, pesquisa aberta, } \\
\text { segundo modelo organizado } \\
\text { de Weick, com } 135 \\
\text { enfermeiros. }\end{array}$ & $\begin{array}{l}\text { Circunstâncias geradoras de conflitos: Comunicação não participativa; } \\
\text { falta de autonomia profissional e de envolvimento nas decisões de cuidado } \\
\text { ao paciente; sobrecarga de trabalho; mensagens culpando enfermeiros por } \\
\text { condições potencialmente fora de seu domínio; comunicação desrespeitosa } \\
\text { e má administração de conflitos prévios. } \\
\text { Sujeitos envolvidos: Enfermeiros e médicos. } \\
\text { Consequências: Conflito mal gerenciado; enfraquecimento das relações } \\
\text { interpessoais; ambiente de trabalho insalubre e frustrante; surgimento de } \\
\text { novos conflitos. }\end{array}$ \\
\hline 2 & $\begin{array}{l}\text { Kim WS, et al. } \\
(2015) \\
\text { Inglaterra }\end{array}$ & $\begin{array}{c}\text { Journal of } \\
\text { Advanced Nursing }\end{array}$ & $\begin{array}{l}\text { Examinar as percepções de } \\
\text { enfermeiros a respeito dos conflitos } \\
\text { construtivos e destrutivos e seu } \\
\text { manejo entre enfermeiros. }\end{array}$ & $\begin{array}{lr}\text { Qualitativo, } & \text { pesquisa } \\
\text { descritiva, } & \text { com } 34 \\
\text { enfermeiros. } & \end{array}$ & $\begin{array}{l}\text { Circunstâncias geradoras de conflitos: Falta de comunicação; restrições } \\
\text { de tempo, relacionamentos e trabalho em equipe; diferenças de poder. } \\
\text { Sujeitos envolvidos: Enfermeiro e médico; enfermeiro e enfermeiro. } \\
\text { Consequências: O conflito bem gerenciado contribui para a resolução de } \\
\text { problemas, promove o aumento da colaboração, melhora na comunicação } \\
\text { e traz resultado positivo para o atendimento ao paciente. }\end{array}$ \\
\hline 3 & $\begin{array}{l}\text { Choe K, et al. } \\
(2015) \\
\text { Inglaterra }\end{array}$ & $\begin{array}{c}\text { Journal of } \\
\text { Advanced Nursing }\end{array}$ & $\begin{array}{l}\text { Explorar e compreender o sofrimento } \\
\text { moral na perspectiva de enfermeiros } \\
\text { intensivistas na Coreia e como este } \\
\text { sofrimento é vivenciado por eles. }\end{array}$ & $\begin{array}{l}\text { Qualitativo, entrevistas com } \\
\text { abordagem profunda face-a- } \\
\text { face, com } 14 \text { enfermeiros } \\
\text { intensivistas. }\end{array}$ & $\begin{array}{l}\text { Circunstâncias geradoras de conflitos: Incapacidade de compartilhar } \\
\text { suas opiniões; falta de autoridade; impossibilidade de prestar atendimento } \\
\text { integral devido à escassez de pessoal. } \\
\text { Sujeitos envolvidos: Enfermeiro e médico; enfermeiro e administração do } \\
\text { hospital; enfermeiro e familiares. } \\
\text { Consequências: Sentimento de impotência. }\end{array}$ \\
\hline 4 & $\begin{array}{c}\text { Jiménez-Herrera } \\
\text { MF e Axelsson C } \\
(2015) \\
\text { Inglaterra }\end{array}$ & Nursing Ethics & $\begin{array}{l}\text { Analisar e descrever situações que } \\
\text { podem gerar conflito ético entre } \\
\text { enfermeiros que atuam em } \\
\text { emergências. }\end{array}$ & $\begin{array}{l}\text { Qualitativo, estudo de grupo } \\
\text { focal, com } 16 \text { enfermeiros de } \\
\text { emergência. }\end{array}$ & $\begin{array}{l}\text { Circunstâncias geradoras de conflitos: Falta de autonomia profissional. } \\
\text { Consequências: } O \text { conflito promove sentimentos de angústia, sofrimento, } \\
\text { raiva e impotência. }\end{array}$ \\
\hline 5 & $\begin{array}{l}\text { Falcó-Pegueroles } \\
\text { A, et al. (2015) } \\
\text { Inglaterra }\end{array}$ & Nursing Ethics & $\begin{array}{l}\text { Examinar o nível de exposição ao } \\
\text { conflito ético em enfermeiros de } \\
\text { unidades de cuidados intensivos e } \\
\text { analisar a relação entre este nível e os } \\
\text { tipos de conflito ético e status morais. }\end{array}$ & $\begin{array}{l}\text { Quantitativo, estudo } \\
\text { correlacional descritivo, com } \\
203 \text { enfermeiros de cuidados } \\
\text { intensivos. }\end{array}$ & $\begin{array}{l}\text { Circunstâncias geradoras de conflitos: Identificação da insuficiência do } \\
\text { tratamento analgésico e/ou sedativo administrado, que gera sofrimento ao } \\
\text { paciente; trabalhar com médicos incompetentes; cuidar de pacientes } \\
\text { internados em unidades inapropriadas para o seu atendimento; falha em } \\
\text { manter confidenciais os dados clínicos dos pacientes e administrar } \\
\text { tratamentos e/ou realizar intervenções quando a família não tem } \\
\text { conhecimento dos objetivos, riscos e benefícios clínicos. } \\
\text { Sujeitos envolvidos: Enfermeiro e médico; enfermeiro e familiares dos } \\
\text { pacientes. }\end{array}$ \\
\hline
\end{tabular}




\begin{tabular}{|c|c|c|c|c|c|}
\hline ID & $\begin{array}{l}\text { Autor, ano e país } \\
\text { de publicação }\end{array}$ & Periódico & Objetivos & Método utilizado & Resultados \\
\hline 6 & $\begin{array}{l}\text { Başoğul C e } \\
\text { Özgür G. (2016) } \\
\text { Coreia do Sul }\end{array}$ & $\begin{array}{l}\text { Asian Nursing } \\
\text { Research (Korean } \\
\text { Soc Nurs Sci) }\end{array}$ & $\begin{array}{l}\text { Analisar os níveis de inteligência } \\
\text { emocional e estratégias de gestão de } \\
\text { conflitos de enfermeiros e a } \\
\text { associação entre eles. }\end{array}$ & $\begin{array}{lr}\text { Quantitativo, } & \text { estudo } \\
\text { transversal e descritivo, com } \\
277 \quad \text { enfermeiros } \\
\text { selecionados em amostra } \\
\text { aleatória. }\end{array}$ & $\begin{array}{l}\text { Circunstâncias geradoras de conflitos: Condições de trabalho; } \\
\text { desequilíbrios de autoridade e poder; diferenças nos critérios e objetivos } \\
\text { profissionais; comunicação inadequada. } \\
\text { Sujeitos envolvidos: Enfermeiros que trabalham na mesma unidade; } \\
\text { enfermeiro e médico; enfermeiro assistencial e enfermeiro gerente de } \\
\text { enfermagem; enfermeiro e auxiliares de enfermagem; enfermeiros de outras } \\
\text { unidades. }\end{array}$ \\
\hline 7 & $\begin{array}{l}\text { Sharma J, et al. } \\
\text { (2016) } \\
\text { EUA }\end{array}$ & $\begin{array}{l}\text { Applied Nursing } \\
\text { research: ANR }\end{array}$ & $\begin{array}{l}\text { Examinar até que ponto os conflitos } \\
\text { trabalho-família causam estresse } \\
\text { entre a equipe de enfermagem e seu } \\
\text { subsequente impacto em sua saúde } \\
\text { psicológica; examinar se o nível de } \\
\text { inteligência emocional da } \\
\text { enfermagem atuou como moderador } \\
\text { entre o nível de estresse dos } \\
\text { profissionais e a saúde psicológica. }\end{array}$ & $\begin{array}{lrr}\text { Quantitativo, } & \text { estudo } & \text { com } \\
\text { análise de } & \text { regressões } \\
\text { múltiplas, } & \text { com } & 693 \\
\text { enfermeiros } & \text { de } & 33 \\
\text { instituições de saúde } & \text { em } \\
\text { Utaracanda (Índia). }\end{array}$ & $\begin{array}{l}\text { Sujeitos envolvidos: Enfermeiros e familiares. } \\
\text { Consequências: Afetou negativamente a saúde psicológica dos } \\
\text { profissionais enfermeiros e causou estresse neles. }\end{array}$ \\
\hline 8 & $\begin{array}{l}\text { Nitzsche A, et al. } \\
(2017) \\
\text { Inglaterra }\end{array}$ & $\begin{array}{l}\text { International } \\
\text { Journal of Nursing } \\
\text { studies Advances }\end{array}$ & $\begin{array}{l}\text { Investigar a ligação entre o capital } \\
\text { social organizacional e conflito } \\
\text { trabalho-casa em profissionais de } \\
\text { saúde. }\end{array}$ & $\begin{array}{lr}\text { Quantitativo, } & \text { estudo } \\
\text { transversal, com } 1.733 \\
\text { enfermeiros. }\end{array}$ & $\begin{array}{l}\text { Circunstâncias geradoras de conflitos: Alta carga de trabalho; baixa } \\
\text { autonomia em relação à programação e métodos de trabalho; baixo apoio } \\
\text { social de supervisores e colegas de trabalho. } \\
\text { Sujeitos envolvidos: Enfermeiros e familiares do profissional (conflito } \\
\text { familiar). }\end{array}$ \\
\hline 9 & $\begin{array}{l}\text { Alazzam M, et al. } \\
\text { (2017) } \\
\text { EUA }\end{array}$ & Nursing Forum & $\begin{array}{l}\text { Explorar a incidência dos conflitos } \\
\text { trabalho-família e a associação entre } \\
\text { estes e o nível de satisfação entre } \\
\text { enfermeiros jordanianos. }\end{array}$ & $\begin{array}{lr}\text { Quantitativo, } & \text { estudo } \\
\text { transversal, com amostra } \\
\text { conveniente de } 333 \\
\text { enfermeiros jordanianos. }\end{array}$ & $\begin{array}{l}\text { Sujeitos envolvidos: Enfermeiros e familiares do profissional (conflito } \\
\text { familiar). }\end{array}$ \\
\hline 10 & $\begin{array}{l}\text { Saberi Z, et al. } \\
(2019) \\
\text { Inglaterra }\end{array}$ & Nursing Ethics & $\begin{array}{l}\text { Analisar a relação do conflito ético } \\
\text { com características pessoais e } \\
\text { organizacionais entre enfermeiros de } \\
\text { cuidados intensivos. }\end{array}$ & $\begin{array}{l}\text { Quantitativo, estudo } \\
\text { correlacional, descritivo, com } \\
216 \quad \text { enfermeiros } \\
\text { selecionados aleatoriamente. }\end{array}$ & $\begin{array}{l}\text { Circunstâncias geradoras de conflitos: Trabalhar com enfermeiras ou } \\
\text { auxiliares de enfermagem profissionalmente incompetentes; trabalhar com } \\
\text { médicos incompetentes; administrar tratamentos de acordo com os desejos } \\
\text { da família sem permissão do paciente; falha na confidencialidade de dados } \\
\text { clínicos dos pacientes; estrutura organizacional e regulamentos } \\
\text { inadequados; sistema de recompensa ineficaz; conduta gerencial e cultura } \\
\text { organizacional inadequadas. } \\
\text { Sujeitos envolvidos: Enfermeiros e enfermeiros; enfermeiros e médicos; } \\
\text { enfermeiros e familiares. }\end{array}$ \\
\hline
\end{tabular}

Fonte: Lima LN de, et al., 2021. 


\section{DISCUSSÃO}

Os artigos analisados foram publicados em idioma inglês, predominantemente em periódicos da Inglaterra e Estados Unidos. Isto pode ser justificado devido o inglês ser o idioma oficial para a comunicação científica e as sedes das principais revistas científicas em saúde no mundo estarem localizadas naqueles países. Em pesquisa que verificou a métrica das publicações em saúde ao longo de 25 anos, constatou-se que os quatro periódicos mais citados estavam nos Estados Unidos ou Inglaterra. Os resultados sugeriram, todavia, que 0 fator país não é levado em consideração no momento da decisão da submissão do trabalho, mas sim a qualidade do periódico. Estes países historicamente concentram os mais bem conceituados periódicos em saúde (MERIGÓ JM e NÚÑES A, 2016).

A maioria dos artigos seguiu a abordagem quantitativa, com média de 575,8 participantes por estudo. Ainda que o tema conflito necessite de investigações qualitativas - abordagem que auxilia a melhor compreender o fenômeno -, parece haver uma tendência, observada ao longo dos anos, quanto ao desenvolvimento de estudos quantitativos nesta área (Quadro 1). O número de participantes por estudo foi significativo para análises estatísticas realizadas, o que indica uma preocupação de pesquisadores da enfermagem para observar os parâmetros estatísticos, almejando resultados mais consistentes. A média de participantes por pesquisa qualitativa esteve acima do recomendado, sendo que um estudo avaliou 135 pessoas (MORELAND JJ e APKER J, 2015), amostra não usual para esse tipo de investigação (SAWATSKY AP, et al., 2019).

Os dados qualitativos foram agrupados em três categorias temáticas: circunstâncias geradoras de conflitos, sujeitos envolvidos, e consequências (dos conflitos). Dentre as circunstâncias geradoras de conflitos, verificou-se que conflitos ocorrem devido a problemas de comunicação. Em estudo realizado em uma emergência hospitalar, problemas ou falhas na comunicação foram verificados principalmente nas situações de troca de turnos e rotatividade de pessoal. Trocas de turno apressadas - no contexto de dupla jornada de trabalho ou de passagem de plantão em grupo - dificultou a compreensão apropriada do quadro clínico do paciente. A alta rotatividade de profissionais, dentre estes, estudantes, também contribui para o distanciamento nas interações interpessoais, tornando o trabalho mais mecânico e pouco humanizado (MARTINS CCF, et al., 2014).

A comunicação, como competência básica para a colaboração interprofissional, está no centro da interação interpessoal e é amplamente explorada no contexto da pesquisa científica e incentivada nos ambientes de trabalho (IPEC, 2016). Problemas de comunicação podem ser prejudiciais ao planejamento dos cuidados clínicos, intensificar situações desarmônicas entre os entes envolvidos, promover descontentamentos, favorecer o desenvolvimento de um ambiente laboral tenso e, consequentemente, comprometer os cuidados de saúde ao paciente (LIMA AWS, et al., 2020).

Problemas na estrutura e/ou ambiente laboral das instituições também foram identificados como circunstâncias geradoras de conflitos. As unidades de urgência e emergência, atualmente organizadas em Redes de Atenção às Urgências e Emergências, são estruturadas com a finalidade de acolher o paciente e estabilizar o seu quadro clínico, podendo, assim, referenciá-lo para outros serviços, seja da rede básica ou especializada de saúde. A falta de estrutura e/ou escassez de insumos compromete a prestação adequada destes serviços (BRASIL, 2013).

Em pesquisa que investigou os fatores associados à intenção dos enfermeiros de permanecer atuando em uma instituição, observou-se que questões relacionadas à organização do serviço foram fundamentais para a decisão de permanecer ou não, especialmente quando estas questões dizem respeito a políticas organizacionais, supervisão, apoio, capacitação e satisfação do profissional (NEED, 2006). Atualmente, nos Estados Unidos, verifica-se uma taxa de $16,5 \%$ de rotatividade de enfermeiros cadastrados nos serviços de saúde, resultando em uma perda estimada entre US\$ 4.21 e US\$ 6.02 milhões para os hospitais. Sugeriu-se que o ambiente positivo (camaradagem entre profissionais, apoio e incentivos por parte da administração) atuou como principal facilitador para reduzir tais perdas (YARBROUGH S, et al., 2016).

A sobrecarga de trabalho foi outro fator relacionado às circunstâncias geradoras de conflito. $A$ este respeito, pesquisa revelou que o setor de emergência possui uma grande demanda de trabalho e que os 
conflitos interpessoais promovem tensão, cansaço e afetam o estado emocional dos profissionais, em especial dos enfermeiros (DUARTE MLC, et al., 2018). A sobrecarga de trabalho, embora não estatisticamente significativa, esteve relacionada a mortalidade de pacientes em uma unidade de cuidados intensivos (KIEKKAS P, et al., 2008). Apesar de não ter havido a associação significativa entre as variáveis, os autores sugeriram que o resultado foi notável. Já resultados de revisão sistemática sugerem que, em todas as especialidades da enfermagem, o acúmulo de tarefas é visto como estressante e, consequentemente, fator de risco para problemas de saúde, como a exaustão e a hipertensão arterial sistêmica (LIM J, et al., 2010).

A segunda categoria temática disse respeito aos sujeitos envolvidos nos conflitos. A análise dos resultados permitiu inferir que há uma predominância da enfermagem quanto ao interesse pela pesquisa sobre conflitos na prática profissional em saúde e que os profissionais da enfermagem tendem a sofrer mais com os conflitos gerados no ambiente de trabalho (Quadro 1). A literatura aponta que, dentre os profissionais da saúde, a equipe de enfermagem apresenta maior risco para vivenciar os conflitos porque, dada a natureza do seu trabalho e a quantidade de pessoas envolvidas, convive por mais tempo com pacientes, familiares e demais membros da equipe de saúde, sendo que esta relação de convivência ocorre, geralmente, em um agitado contexto de vida e morte, poder e submissão (AMARAL EMS, et al., 2017; SAUER PA e MCCOY TP, 2017).

Esta revisão identificou que os conflitos se deram mais entre enfermeiros e familiares do paciente. Verificou-se, também, a necessidade de estudos que averiguem os motivos pelos quais esses conflitos ocorrem visto que, durante a busca na literatura para a realização desta pesquisa, não foram encontrados resultados que esclarecessem esses motivos.

Resultados de estudos em outros ambientes de saúde podem ajudar a compreender o fenômeno supracitado. Por exemplo, uma pesquisa listou três potenciais barreiras que resultaram em conflitos entre familiares e enfermeiros cuidadores de idosos. A primeira foi o não reconhecimento da expertise do cuidador, quando o familiar tem dificuldade de compreender e aceitar que o profissional da saúde possui as habilidades necessárias para promover o cuidado. A segunda foi a sobreposição do papel de cuidador, quando o familiar deseja ajudar ou mesmo realizar por si só os cuidados ao paciente. A terceira foram as deficiências da própria instituição - déficit de recursos humanos, escassez de insumos e problemas de infraestrutura - que afetam a qualidade do trabalho dos profissionais atuantes e transmitindo, para o familiar, a ideia de que o paciente não está sendo bem cuidado (KONNERT C, et al., 2017).

Conflitos entre enfermeiros e a equipe médica também ocorrem com frequência. Dentre todo o corpo de profissionais da saúde, a enfermagem parece estar mais vulnerável devido ao assédio moral a que está sujeita. Há registro de estudos sobre assédio moral sofrido por profissionais da enfermagem desde, pelo menos, a década de 1980 e a ocorrência deste tipo de assédio tem contribuído negativamente para o desempenho das funções em equipe. Consequentemente, foram observados, em estudo, o surgimento de dificuldades quanto ao compartilhamento de informações a respeito do paciente, aumento das taxas de absenteísmo e de desistência da profissão. Estes resultados sugerem que níveis elevados de estresse, efeitos negativos sobre a saúde mental, sentimento de reclusão e sufocamento laboral são comuns em profissionais que experienciam o assédio moral e outros problemas relacionados (SAUER PA e MCCOY TP, 2016).

As funções do médico e do enfermeiro, embora distintas, por vezes são confundidas. Além disso, enfermeiros julgam que a divisão inadequada das atribuições da medicina e da enfermagem, nos serviços de saúde, pode atrapalhar e sobrecarregar o desempenho da equipe de enfermagem (SANTOS OS, et al., 2015). Estudo que objetivou compreender os conflitos vivenciados pelos enfermeiros e as subsequentes estratégias para o seu enfrentamento constatou que o enfermeiro se sentia como uma espécie de secretário de médico, desperdiçando o tempo, que seria destinado aos cuidados de enfermagem, com o preenchimento de documentos de responsabilidade do médico (AMESTOY SC, et al., 2014).

Equipes de enfermagem e médicas precisam ter suas competências bem esclarecidas entre si visando, dentre outros, a promoção de um ambiente agradável de trabalho em equipe, a otimização do tempo, a economia e evitação de desperdício de recursos, a melhoria do custo-benefício nos procedimentos em saúde e a disponibilidade da melhor assistência que o serviço possa oferecer. As academias, as gestões de serviços, 
os órgãos representantes e fiscalizadores de classe podem auxiliar os profissionais da equipe multidisciplinar quanto ao reconhecimento de suas atribuições privativas e daquelas realizadas enquanto equipe de saúde (AL-HAMDAN Z, et al., 2016; BESERRA EP, et al., 2018).

Relatos de conflitos entre os profissionais do próprio corpo da enfermagem foram identificados e os principais motivos foram dificuldades de relacionamento, dificuldades na relação de autoridade e poder, escassez de recursos humanos e profissionais pouco habilitados para o serviço. Em pesquisa, verificou-se que conflitos entre a equipe de enfermagem ocorreram como consequência da falta de compromisso com 0 trabalho, as divergências quanto à conduta a ser adotada, à falta de diálogo entre a equipe, à difusão de boatos no ambiente de trabalho e, até mesmo, à definição das escalas de serviço. Por se tratar de uma equipe dinâmica, com alta rotatividade de pessoal, que precisa lidar com as tarefas mais árduas da prestação dos cuidados e fazer cooperar profissionais com posições hierárquicas distintas, a convivência harmônica se torna um objetivo a ser constantemente alcançado e mantido (SPAGNOL CA, et al., 2010).

A terceira categoria temática referiu-se às consequências dos conflitos existentes. A enfermagem, mais uma vez, ganha protagonismo porque tende a arcar mais com as consequências das relações conflituosa. $O$ estresse é o primeiro sinal consequente dos conflitos, interfere nas relações familiares do enfermeiro e afeta a sua saúde mental (SHARMA J, et al., 2016). Não raros, os sentimentos de inferioridade são manifestados por meio de atitudes de reclusão, sensação de sufocamento (resultante da sobrecarga laboral) e baixa autoestima para atuar ou desenvolver habilidades para a melhoria da qualidade da assistência (CHOE K, et al., 2015; JIMÉNEZ-HERRERA MF e AXELSSON C, 2015; MORELAND JJ e APKER J, 2015; SHARMA J, et al., 2016). Somado a isso, enfermeiros sofrem com o sofrimento de pacientes, principalmente em ocasiões em que, dada a impotência para a tomada de certas decisões, estes profissionais nada podem fazer para ajudar (FALCÓ-PEGUEROLES A, et al., 2015).

Naturalmente, pode-se inferir que as consequências dos conflitos são necessariamente negativas. Porém, nesta revisão, verificou-se que respostas positivas foram dadas mediante a competente gestão das crises. $\mathrm{A}$ resolução de todos os conflitos ou mesmo a inexistência destes no ambiente de saúde é um vislumbre utópico. Isto porque o conflito é inerente à existência humana e resulta da natureza divergente das visões de mundo entre as pessoas e de problemas relacionados ao funcionamento dos serviços. Entende-se que o serviço de saúde pode crescer em meio aos conflitos, e é a resposta da gestão a esses conflitos, ou seja, a maneira como a administração os gerencia, que definirá se o impacto da crise produziu mais efeitos positivos ou negativos (MORELAND JJ e APKER J, 2015; KIM WS, et al. 2015).

Estudo mostrou que o sistema de gestão preferido pelos enfermeiros era o integrativo, em que há a busca de uma solução aceitável para todos, seguido da transigência, isto é, atitude intermediária aceitável para ambas as partes. Já o sistema menos usado era o dominante, em que o alcance dos objetivos de uma das partes é considerado prioritário perante os interesses da outra parte (AL-HAMDAN Z, et al., 2016). Nesse estudo, os autores sugerem que, para culturas coletivistas, onde há a priorização dos objetivos do grupo, como é o caso do Brasil, o estilo integrativo pode ser um bom sistema de gestão de conflitos a ser adotado. A apropriada gestão dos conflitos, seja entre profissionais e familiares, entre profissionais de classes distintas, ou de mesma classe, deve ser parte integrante dos espaços hospitalares, pois a gestão prudente promove resultados positivos em todos os aspectos (BESERRA EP, et al., 2018).

\section{CONSIDERAÇÕES FINAIS}

A existência de conflitos é uma realidade em ambientes de urgência e emergência e ocorrem como resultante das dificuldades de comunicação, infraestrutura inadequada dos serviços e sobrecarga de trabalho. Todavia, entende-se que é possível extrair lições positivas dos conflitos, as quais podem contribuir para o aperfeiçoamento das relações no ambiente de trabalho. Alerta-se para que a gestão dos serviços atente para os conflitos envolvendo profissionais da enfermagem, classe profissional mais vulnerável. As limitações para a realização desta revisão estiveram relacionadas à escassez de estudos sobre o tema nas bases de dados pesquisadas, às diversidades culturais na prática profissional nos diferentes países - o que dificultou a síntese dos resultados - e a ausência de estudos que descrevem a realidade de países pobres ou emergentes, onde a carência de profissionais é acentuada e a sobrecarga de trabalho é extenuante. 


\section{REFERÊNCIAS}

1. AL-HAMDAN Z, et al. Conflict management style of Jordanian nurse managers and its relationship to staff nurses' intent to stay. J Nurs Manag, 2016; 24(2): E137-45.

2. ALAZZAM M, et al. The relationship between work-family conflict and job satisfaction among hospital nurses. Nurs Forum, 2017; 52(4): 278-288.

3. AMARAL EMS, et al. Perceptions about the work of the nursing team in adult emergency hospital service. Rev Min Enferm, 2017; 21: e-1023.

4. AMESTOY SC, et al. Conflict management: challenges experienced by nurse-leaders in the hospital environment. Rev Gaúcha Enferm., 2014; 35(2): 79-85.

5. BAŞOĞUL C, ÖZGÜR G. Role of emotional intelligence in conflict management strategies of nurses. Asian Nurs Res, 2016; 10(3): 228-233.

6. BESERRA EP, et al. Conflict management in nursing training. J Nurs UFPE online, 2018; 12(10): 2891-2896.

7. BRASIL. Ministério da Saúde. Secretaria de Atenção à Saúde. Departamento de Atenção Especializada. Manual instrutivo da Rede de Atenção às Urgências e Emergências no Sistema Único de Saúde (SUS). Brasília: Editora do $\begin{array}{llllll}\text { Ministério da } & \text { Saúde, } & 2013 ; & 84 & \text { p. } & \text { Disponível }\end{array}$ https://bvsms.saude.gov.br/bvs/publicacoes/manual_instrutivo_rede_atencao_urgencias.pdf. Acessado em: 28 de julho de 2021.

8. CHOE K, et al. Moral distress in critical care nurses: a phenomenological study. J Adv Nurs, 2015; 71(7): $1684-1693$.

9. DUARTE MLC, et al. Work in hospital emergency: suffering and defensive nursing care strategies. Rev Gaúcha Enferm, 2018; 39: e2017-0255.

10. FALCÓ-PEGUEROLES A, et al. Ethical conflict in critical care nursing: correlation between exposure and types. Nurs Ethics, 2015; 22(5): 594-607.

11. FERNANDES RTP, COELHO MJ. Superlotação de emergências: um novo cenário para o cuidar/cuidado em enfermagem. Rev Eletrôn Estácio Saúde, 2013; 2(1): 19-23.

12. FROTA CA, et al. Dificuldades enfrentadas pelos enfermeiros na realização da classificação de risco no serviço de urgência e emergência. Revista Eletrônica Acervo Saúde, 2021; 13(2): e5498.

13. IPEC. Interprofessional Education Collaborative. 2016. Core competencies for interprofessional collaborative practice: 2016 update. Washington, DC: Interprofessional Education Collaborative. Available from: https://aamcmeded.global.ssl.fastly.net/production/media/filer_public/70/9f/709fedd7-3c53-492c-b9f0b13715d11cb6/core_competencies_for_collaborative_practice.pdf. Cited Apr 07, 2021.

14. JIMÉNEZ-HERRERA MF, AXELSSON C. Some ethical conflicts in emergency care. Nurs Ethics, 2015; 22(5): 548560.

15. KIEKKAS $P$, et al. Association between nursing workload and mortality of intensive care unit patients. $J$ Nurs Scholarsh, 2008; 40(4): 385-90.

16. KIM WS, et al. Nurses' perceptions of conflict as constructive or destructive. J Adv Nurs, 2015; 71(9): $2073-2083$.

17. KONNERT C, et al. Conflict between family caregivers and staff in nursing homes: feasibility of the daily diary method. Clin Gerontol, 2017; 40(5): 352-361.

18. LANZ JJ, BRUK-LEE V. Resilience as a moderator of the indirect effects of conflict and workload on job outcomes among nurses. J Adv Nurs, 2017; 73(12): 2973-2986.

19. LIMA AWS, et al. Perception and manifestation of collaborative competencies among undergraduate health students. Rev. Latino-Am. Enfermagem, 2020; 28: e3240.

20. LIM J, et al. Stress and coping in Australian nurses: a systematic review. Int Nurs Rev, 2010; 57(1):22-31.

21. MARTINS CCF, et al. The nursing team's interpersonal relationships v. stress: limitations for practice. Cogitare Enferm, 2014; 19(2): 287-293.

22. MERIGÓ JM, NÚNEZ A. Influential journals in health research: a bibliometric study. Global Health, $2016 ; 12(1): 46$.

23. MORELAND JJ, APKER J. Conflict and stress in hospital nursing: improving communicative responses to enduring professional challenges. Health Commun, 2016; 31(7): 815-823.

24. NEED N. Perceptions of empowerment and intent to stay. Nurs Econ., 2006; 24(1): 13-8,3.

25. NITZSCHE A, et al. Staff working in hospital units with greater social capital experience less work-home conflict: secondary analysis of a cross-sectional study. Int J Nurs Stud, 2017; 75: 139-146.

26. PICOLI RP, et al. Service users classified as risk level 'blue' in an emergency department. Cogitare Enferm, 2016; 21(1): 01-07.

27. SABERI Z, et al. The relationship between ethical conflict and nurses' personal and organisational characteristics. Nurs Ethics, 2019; 26(7-8): 2427-2437.

28. SANTOS PS, et al. Relação entre médicos e enfermeiros do Hospital Regional de Cáceres Dr. Antônio Fontes: a perspectiva do enfermeiro. Rev Ciênc Estud Acad Med, 2015; 4: 10-28.

29. SAUER PA, MCCOY TP. Nurse Bullying: Impact on Nurses' Health. West J Nurs Res, 2017; 39(12): $1533-1546$.

30. SAWATSKY AP, et al. Qualitative research methods in medical education. Anesthesiology, 2019; 131(1): 14-22.

31. SHARMA J, et al. Stress as a mediator between work-family conflict and psychological health among the nursing staff: moderating role of emotional intelligence. Appl Nurs Res, 2016; 30: 268-275.

32. SPAGNOL CA, et al. Conflict situations experienced at hospital: the view of nursing technicians and auxiliaries. Rev Esc Enferm USP, 2010; 44(3): 792-9.

33. VIVAR CG. Putting conflict management into practice: a nursing case study. J Nurs Manag, 2006; 14(3): 201-206.

34. WHITTEMORE R, KNALF K. The integrative review: update methodology. J Adv Nurs, 2005; 52(5): 546-553.

35. YARBROUGH S, et al. Professional values, job satisfaction, carrer development, and intent to stay. Nurs. Ethics, $2016 ; 24(6): 675-685$. 